\title{
On the use of computational multi-body dynamics analysis in SLS-based 3D printing
}

\author{
Hammad Mazhar ${ }^{\mathrm{a}}$, Tim Osswald ${ }^{\mathrm{b}}$, Dan Negrut ${ }^{\mathrm{a}, *}$ \\ ${ }^{a}$ Simulation-Based Engineering Lab, Dept. of Mechanical Engineering, University of Wisconsin-Madison, Madison, Wisconsin 53706 \\ ${ }^{b}$ Polymer Engineering Center, Dept. of Mechanical Engineering, University of Wisconsin-Madison, Madison, Wisconsin 53706
}

\begin{abstract}
In the context of additive manufacturing, we illustrate how computational multi-body dynamics (CMBD) analysis can (a) increase printing throughput; and, (b) play a role in improving the quality of 3D printed parts. Throughput is increased by packing the printing volume with as many parts as possible. The problem becomes one of determining where each component that needs to be printed finds itself inside the printing volume. Finding the position and orientation of each part is accomplished through CMBD analysis, a point illustrated through an example in which an open-source dynamics engine called Chrono is used to simulate the filling of the active printing volume with a dress that is subsequently 3D printed. This approach, which is general in purpose, enables one to print in one pass multiple parts that are virtually; i.e., through simulation, dropped inside the printing volume to fully fill it up, thus improving efficiencies. In relation to (b), we use million-body dynamics simulations to gauge how various granular mixture parameters and rolling regimes combine to ultimately control the roughness of the surface being sintered.
\end{abstract}

Keywords: Computational Multibody Dynamics, Improving Throughput, Simulation, Selective Laser Sintering, Additive Manufacturing, Parallel Computing

\section{Background}

\subsection{Rapid Prototyping and $3 D$ Printing}

Additive manufacturing processes allow 3D objects of almost arbitrary geometry to be created by layering material of different types. There are several methods used for 3D printing today, e.g., selective laser melting (SLM), selective laser sintering (SLS), direct metal laser sintering (DMLS), stereolithography, and fused deposition modeling (FDM) [1, 2, 3, 4]. Techniques such as SLS and DMLS allow 3D parts to be generated by solidifying successive layers of material [5]. Additive manufacturing has shown impressive growth in the last two decades. SLS in particular, owing to its ability to work with many different types of materials, has been one of the fastest growing additive manufacturing techniques [6].

Today, additive manufacturing is used in many industries owing to a drop in prices for 3D FDM printers and a strong hobbyist community that has generated a large body of open source designs that are 3D printing ready. In the aerospace industry, SpaceX and NASA are both investigating how laser sintering can improve rocket engine performance [7, 8]. NASA has sent a 3D printer to the International Space Station and recently printed a wrench in anticipation of future missions to Mars in which astronauts will print their own tools and replacement parts $[9,10,11]$.

For additive manufacturing to be adopted in the mainstream manufacturing of goods, high 3D printing throughput is mandatory. For instance, given the task of 3D printing a special washer

\footnotetext{
${ }^{*}$ Corresponding Author.

Email address: negrut@wisc.edu (Dan Negrut)
}

design, it would be extremely inefficient to print one washer at a time, or for that matter a collection of washers that are lying at the bottom of the printing volume. Instead, one should consider filling the entire print volume with washers that are slightly separated. Specifying where each washer is positioned in the volume is straightforward - owing to its simple geometry one can work out by hand the position of each washer in the print volume and use that position and the known washer geometry to program the printer. Then, in one full pass, the printer can generate a large number of washers. This idea of filling up the print volume with parts that need to be $3 \mathrm{D}$ printed is appealing, yet its applicability is limited by one's ability to decide where each part is positioned and how it is oriented. Indeed, when printing a more complex assembly like a chain-mail dress, for instance, the dress will have to be folded, in which case determining the location of each component poses a nontrivial question. This question can be answered via CMBD simulation.

SLS additive manufacturing, carried out on a device shown in Fig. 1, has a somewhat limited range of applications due to challenges in reproducibility of parts. For instance, the influence of the process conditions on the dimensional accuracy is not well understood, which can compromise the quality of 3D printed parts. Indeed, the impact of the powder packing density and geometry need to be studied further to quantify their net effect on the surface quality and porosity of the final part [12]. To this end, we propose to use large scale CMBD analysis to understand how the shape of the particles, the nature of the mutual frictional contact interaction between the different materials being mixed together, and the rolling regimen; i.e., roller translation vs. rotation speeds, influence the outcome of the rolling process, which is ultimately dictating the quality of 


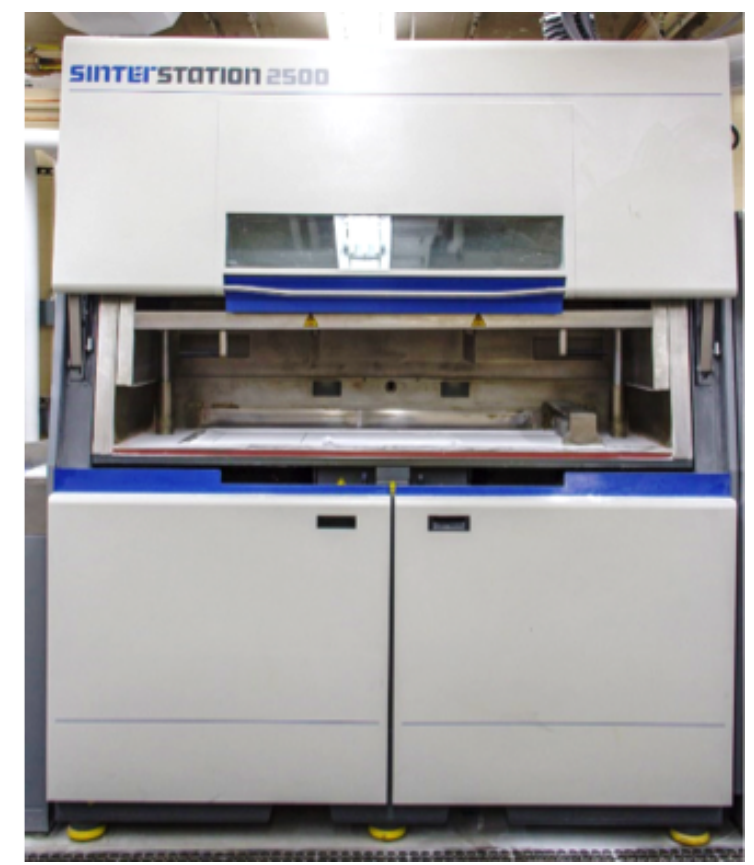

Figure 1: The Sinter Station 2500 used for 3D printing

the manufactured part.

This document is organized as follows. Section $§ 1.2$ introduces the set of Newton-Euler equations of motion that govern the time evolution of systems of rigid and flexible bodies. The equations of motion account for the frictional contact forces acting at the interface between mutually interacting bodies. Section $\S 1.3$ discusses an open source dynamics engine, called Chrono, used herein to solve the aforementioned Newton Euler equations of motion. The process followed to manufacture a dress, which is folded and 3D printed inside a small printer volume, is discussed in $\S 2$. Section $\S 3$ illustrates Chrono's use in the analysis of a rolling process, which calls for solving millions of equations of motion. The paper concludes with a summary of this contribution's main points and a set of directions of future work.

\subsection{Computational Multibody Dynamics Basics}

The dynamics of articulated systems composed of rigid and flexible bodies is characterized by a system of index 3 Differential Algebraic Equations (DAEs) [13, 14, 15] shown using the terms in black font in Eqs. (1a)-(1d):

The differential equations in (1a) relate the time derivative of the generalized positions $\mathbf{q}$ and velocities $\mathbf{v}$ through a linear transformation defined by $\mathbf{L}(\mathbf{q})$. The force balance equation in (1c) ties the inertial forces to the applied and constraint forces, $\mathbf{f}(t, \mathbf{q}, \mathbf{v})$ and $-\mathbf{g}_{\mathbf{q}}^{\mathrm{T}}(\mathbf{q}, t) \hat{\lambda}$, respectively. The latter are imposed by bilateral constraints that restrict the relative motion of the rigid or flexible bodies present in the system. These bilateral constraints, which lead to Eq. (1d), can be augmented by unilateral constraints associated with contact/impact phenomena. To that end, the concept of equations of motion is extended to employ differential inclusions [16]. The simplest example is that of a body that interacts with the ground through friction and contact, when the equations of motion become an inclusion $\mathbf{M} \ddot{\mathbf{q}}-\mathbf{f} \in \mathbf{F}(\mathbf{q}, t)$, where $\mathbf{M}$ is the inertia matrix, $\ddot{\mathbf{q}}$ is the body acceleration, $\mathbf{f}$ is the external force, and $\mathbf{F}(\mathbf{q}, t)$ is a setvalued function. The inclusion states that the frictional contact force lies somewhere inside the friction cone, with a value yet to be determined and controlled by the stick/slip state of the interaction between body and ground. In MBD the differential inclusion can be posed as a differential variational inequality problem [17], which brings along the red-font terms in Eq. (1) [18, 19, 20, 21, 22, 23]. Specifically, the unilateral constraints define a set of contact complementarity conditions $0 \leq \Phi_{i}(\mathbf{q}) \perp \widehat{\gamma}_{i, n} \geq 0$, which make a simple point: for a potential contact $i$ in the active set, $i \in \mathcal{A}(\mathbf{q}(t))$, either the gap $\Phi_{i}$ between two geometries is zero and consequently the normal contact force $\widehat{\gamma}_{i, n}$ is greater than zero, or vice versa. The last equation poses an optimization problem whose first order Karush-KuhnTucker optimality conditions are equivalent to the Coulomb dry friction model [17]. This optimization problem locally maximizes the energy dissipated for a given contact $i$ which for problems with many contacts results in a global maximization of the energy dissipated through the frictional forces. The frictional contact force associated with contact $i$ leads to a set of generalized forces, shown in red in Eq. (1c), which are obtained using the projectors $\mathbf{D}_{i, n}, \mathbf{D}_{i, u}$, and $\mathbf{D}_{i, w}$ [24].

$$
\begin{aligned}
& \dot{\mathbf{q}}=\mathbf{L}(\mathbf{q}) \mathbf{v} \\
& \mathbf{M}(\mathbf{q}) \dot{\mathbf{v}}=\mathbf{f}(t, \mathbf{q}, \mathbf{v})-\mathbf{g}_{\mathbf{q}}^{\mathrm{T}}(\mathbf{q}, t) \hat{\lambda} \\
& +\sum_{i \in \mathcal{F}(\mathbf{q}, \delta)} \underbrace{\left.\widehat{\gamma}_{i, n} \mathbf{D}_{i, n}+\widehat{\gamma}_{i, u} \mathbf{D}_{i, u}+\widehat{\gamma}_{i, w} \mathbf{D}_{i, w}\right)}_{i^{h} \text { frictional contact force }} \\
& \mathbf{0}=\mathbf{g}(\mathbf{q}, t) \\
& i \in \mathcal{A}(\mathbf{q}(t)):\left\{\begin{array}{l}
0 \leq \Phi_{i}(\mathbf{q}) \perp \widehat{\gamma}_{i, n} \geq 0 \\
\left(\widehat{\gamma}_{i, u}, \widehat{\gamma}_{i, w}\right)=\underset{\sqrt{\bar{\gamma}_{i, u}^{2}} \overline{\operatorname{argmin}}_{i, w}^{2} \leq \mu_{i} \bar{\gamma}_{i, n}}{T} \cdot\left(\bar{\gamma}_{i, u} \mathbf{D}_{i, u}+\bar{\gamma}_{i, w} \mathbf{D}_{i, w}\right) .
\end{array}\right.
\end{aligned}
$$

\subsection{Chrono: An Open Source Simulation Engine}

The problem posed in Eq. 1 cannot be solved analytically for any but the most trivial mechanical systems. Therefore, one has to resort to the use of CMBD to produce a numerical solution on a time grid $t_{0}<t_{1}<t_{2}<\ldots<T_{\text {end }}$. There are several open-source dynamics engines available to produce this numerical solution. Bullet [25] is geared towards real-time dynamics analysis of small problems in the context of graphics and video games. Bullet can be used to analyze the dynamics of granular material undergoing frictional contact, soft bodies using spring-damping elements and several different types of joints. ODE [26] provides a rich set of modeling elements with many different types of joints, actuators, and collision models that can be used in real time applications. DART [27] and Simbody [28] support robotics and biomechanics applications, respectively.

Chrono[29], which is the dynamics engine used herein, was designed for large scale mechanical engineering applications. Its forte is handling frictional contact problems, which can be 
solved in either a penalty or a complementarity framework, see Sec. 1.2. Similar to Bullet and ODE, Chrono can be used for expeditious analysis of small problems with tens to hundreds of objects and constraints. Yet Chrono is different in two regards. First, it is extensively validated against experimental data, a process that is ongoing [30, 31, 32, 33, 34]. Second, using the ODE and Bullet engines to solve dynamics problems with millions of rigid bodies requires prohibitively long simulation times, which confines their use to problems of relatively small size. Chrono was designed from ground up to tackle large problems, an attribute reflected in a $\mathrm{C}++$ code structure positioned to leverage parallel computing on Graphics Processing Unit (GPU) cards and multi-core CPUs [35].

For frictional contact problems in a penalty framework, Chrono implements index 3 DAE solutions [36, 37]. For handling frictional contact using a complementarity approach, Chrono implements a variant of the Nesterov algorithm [38]. The modeling of flexible bodies relies on the Absolute Nodal Coordinate Formulation [39]. For Fluid-Solid Interaction (FSI) problems, Chrono uses a smoothed particle hydrodynamics methodology, see [40, 41, 42].

\section{3D Printing Complex Interconnected or Disjoint Struc- tures}

The central idea of this contribution is that CMBD analysis can assist the SLS-based 3D printing process. First, it can increase throughput. Second, it can help with the printing of very complex structures. In both cases, the problem becomes one of determining where each component/part that needs to be printed finds itself inside the printing volume. Finding the position and orientation of each part is accomplished through CMBD analysis. For increasing throughput, consider the task of printing washers. The washer geometry is slightly changed by "inflating" it through a virtual buffering to yield a bufferedpart. The purpose of buffering is to ensure that the actual parts do not touch each other and can be eventually printed without sintering one to another. Buffering can be done through spherical padding or sliding a small sphere all over the surface of the part. The buffered-parts are subsequently dropped into the printing volume up until it is entirely filled up. The true position and orientation of each part is determined by relying on Chrono, which through dynamics analysis determines the end location of each buffered-part. The actual position and orientation of the washer along with its geometry is subsequently passed to the 3D printer, which generates each washer. Note that at any given time, the printer works on generating one layer in many washers. This approach is general in purpose in that it enables one to print in one pass multiple parts that are virtually dropped inside the printing volume to fully fill it, thus improving efficiencies. One might also consider mixing different types of parts, say washers and bolts, to be 3D printed at the same time. Finally, one could shake the box filled with these parts to make them settle and thus add more parts to further increase throughput.

In terms of printing complex structures, the basic idea is illustrated by considering the task of printing a chain-mail dress modeled in Solidworks ${ }^{\circledR}$. Given that the Sinter Station 2500 in Fig. 1 has a build volume which corresponds to a 10 in cube, it is necessary to first compress/fold the dress into this volume. The dropping of the dress into the printing volume is simulated using Chrono. Figure 2 shows the overall process of taking the CAD model, folding the dress using Chrono, and finally converting the settled configuration into a file format readable by the $3 \mathrm{D}$ printer. The idea of using buffered-parts is adopted here as well.

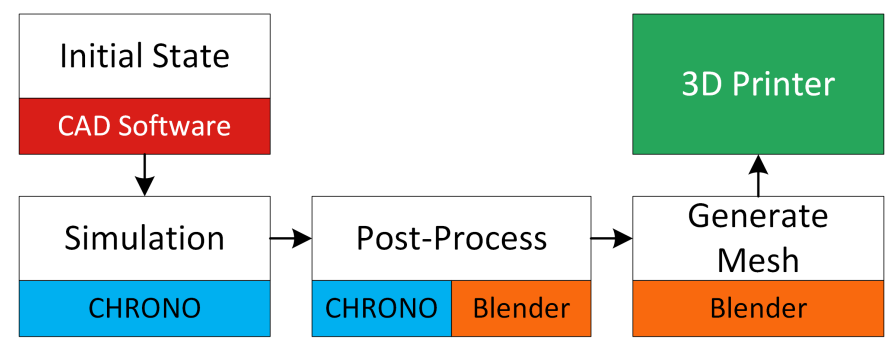

Figure 2: Overall Workflow

Following a chain-mail pattern, the Solidworks ${ }^{\circledR}$ dress was generated adding ring after ring (ring diameter $10 \mathrm{~mm}$; thickness $1 \mathrm{~mm}$ ). The dress was then exported via Chrono::Solidworks [43], a plugin that generates a Chrono CAE model form a Solidworks ${ }^{\circledR}$ CAD model. For the problem at hand, the model consisted of approximately 40000 rings, each represented as a collection of 20 cylinders that combined to generate a buffered-part. The buffered-rings were twice as thick as the actual part. By increasing the thickness of the buffered-rings to $2 \mathrm{~mm}$ during simulation and then reducing it back to $1 \mathrm{~mm}$ for the actual part, the separation between rings was guaranteed to be $1 \mathrm{~mm}$. This separation was required by the $3 \mathrm{D}$ printer to guarantee that individual rings would not fuse together during the sintering process. Simulation was performed on an Intel i7-4770K CPU and was completed in approximately eight hours.

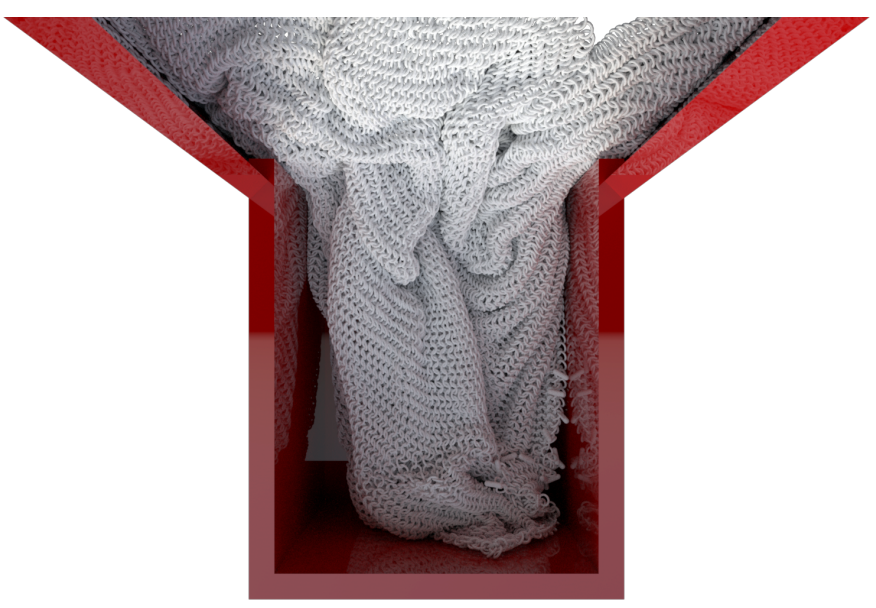

Figure 3: Dress made up of 40000 liked rings falling into the printing volume.

Upon finishing the dynamics analysis in Chrono, the raw position and orientation of the rings had to be converted into 


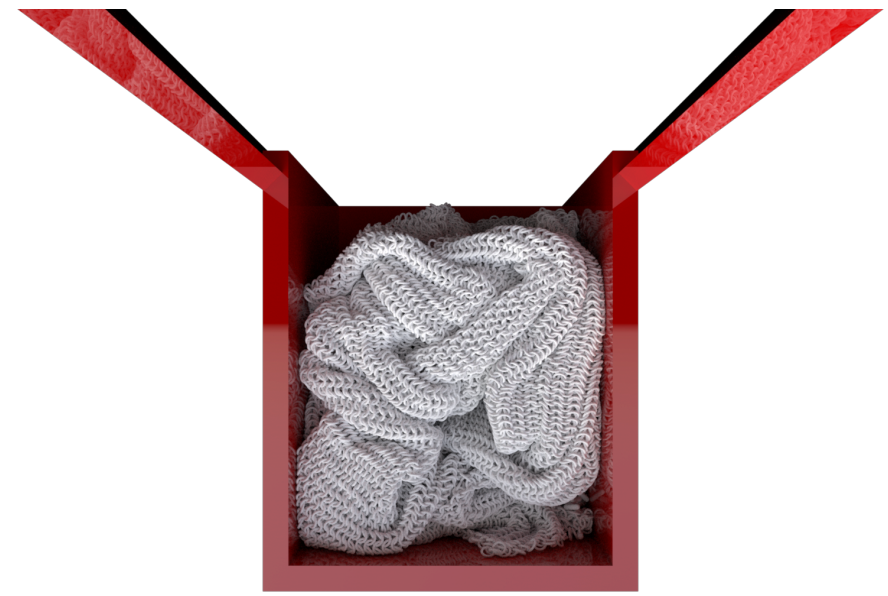

Figure 4: Compressed dress made up of 40000 liked rings.

an STL file required by the 3D printer. This process was done using Blender [44], an open source 3D modeling tool, via its Python application programming interface. Two frames of the

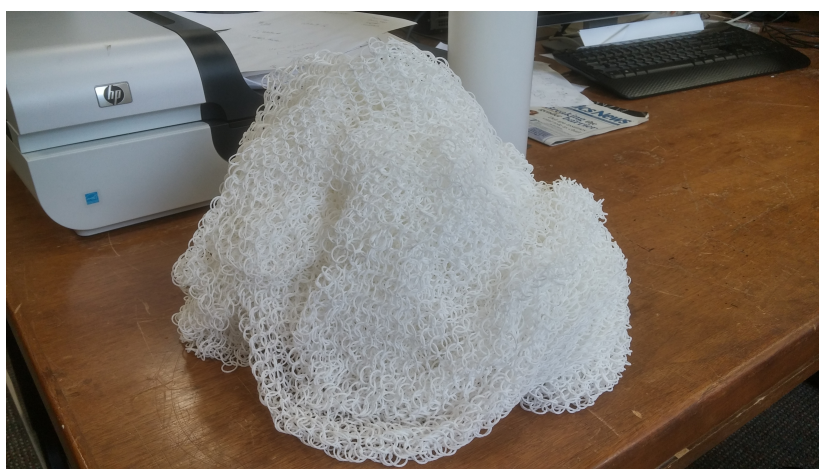

Figure 5: 3D printed dress

Chrono-enabled dynamics analysis are shown in Fig. 3 and Fig. 4, the latter illustrating the settled dress. The dress drops top first under gravity into a $10 \mathrm{inch}^{3}$ box. A square funnel was used to compress the dress into the print volume. The dress was small enough to fit into the build volume under its own weight without additional compression. The final 3D printed dress is shown in Fig. 5. A closeup of the clasps used to close the back of the dress are shown in Fig. 6.

Note that for larger models a heavy block or a plate on a linear actuator can be used in Chrono to slowly press the model into the print volume. Shaking could further improve settling and lead to volume reduction. This aspect is important, since in many additive manufacturing methods the material used for printing is expensive and recycling the unused portion usually compromises the quality of the subsequent parts. Thus, it is advantageous to cram in the printing volume as many parts as possible since this improves both the utilization of the powder and the throughput of the printing process.

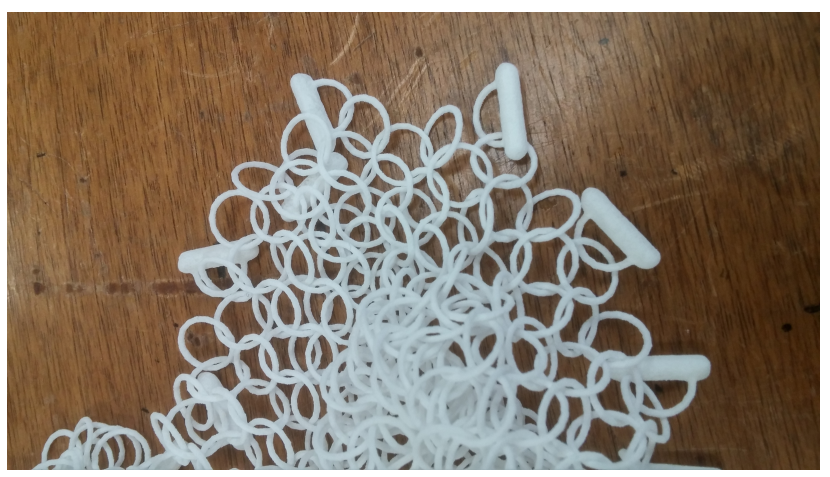

Figure 6: Closeup of clasps in the 3D printed dress

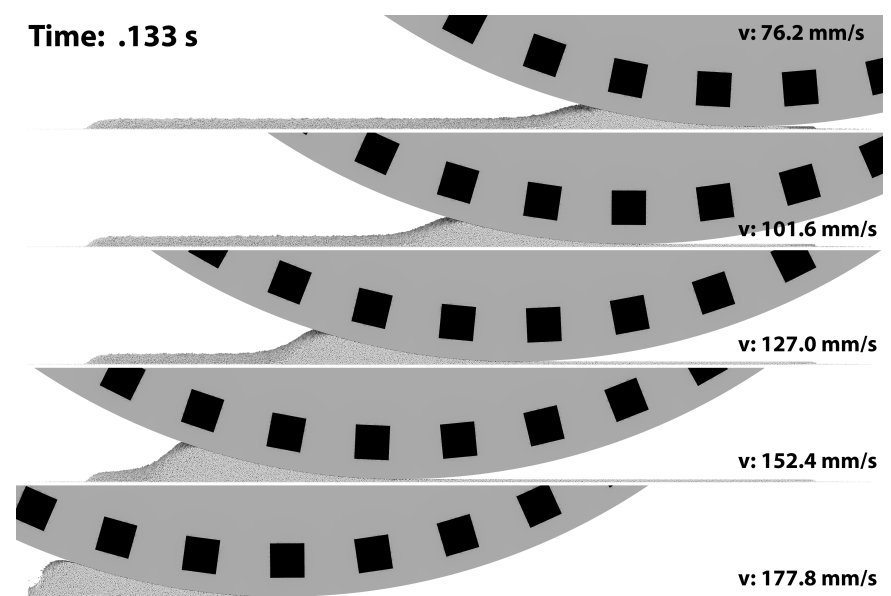

Figure 7: Side view of the SLS simulation at the same time with different roller speeds. The square markers were used to track the rotation of the roller during simulation.

\section{CMBD in Additive Manufacturing}

Many different types of additive manufacturing methods used in industry today, e.g., FDM, SLS, and stereolithography, can be improved through the use of CMBD analysis. CMBD provides $(i)$ insights into how various stages of the manufacturing process work; and, (ii) sensitivities with respect to operating parameters, two pieces of information that can guide efforts to increase throughput and improve part quality. For demonstration purposes, we look at an example in which CMBD is used to characterize the SLS layering of new powder for the Sinter Station 2500 of Fig. 1. The thickness of the new layer and the speed at which it is spread influence the roughness of the powder surface and implicitly the quality of the part. Figure 7 shows side view snapshots taken $0.133 \mathrm{~s}$ into several layering simulations. The roller, which has a diameter of 0.0762 meter, travels at various longitudinal speeds, constant for each simulation, and rotates at a fixed rate of $3.33 \mathrm{rad} / \mathrm{s}$. The salient point made by these snapshots is that translation speed, reported in each snapshot in the lower right corner, influences the shape of the material wave and the powder compaction, which for this example represent the dynamic emergent behavior of a collection of 1300000 rigid spheres of average diameter $55 \mu \mathrm{m}$ and 
density $930 \mathrm{~kg} / \mathrm{m}^{3}$ [32]. The powder particle radii were sampled from a normal distribution. An approximate equation tally for this analysis is as follows: close to eight million from (1a) and the same count for (1c). There are approximately 7.8 million contact complementarity conditions for the normal force and, because the problem accounts for normal, sliding, rolling and spinning of the bodies, there are six Lagrange multipliers for each contact. The optimization problem that provided the frictional contact forces for this simulation was posed in approximately 46.8 million variables and solved in Chrono using parallel computing following a methodology discussed in [38]. These simulations, which currently take approximately one week to complete, are run in a design of experiments setup using at the same time multiple compute nodes. The simulation step size was $0.00001 \mathrm{~s}$, a value dictated by the relative size difference between the powder and the roller on the one hand, and the speed of the roller on the other hand. In effect, this represented a multi-scale problem, given that the radius of the roller and the average radius of the powder were three orders of magnitude apart. Simulation was performed on a machine with $4 \mathrm{x}$ AMD 6274 CPUs and was completed in approximately 6 days.

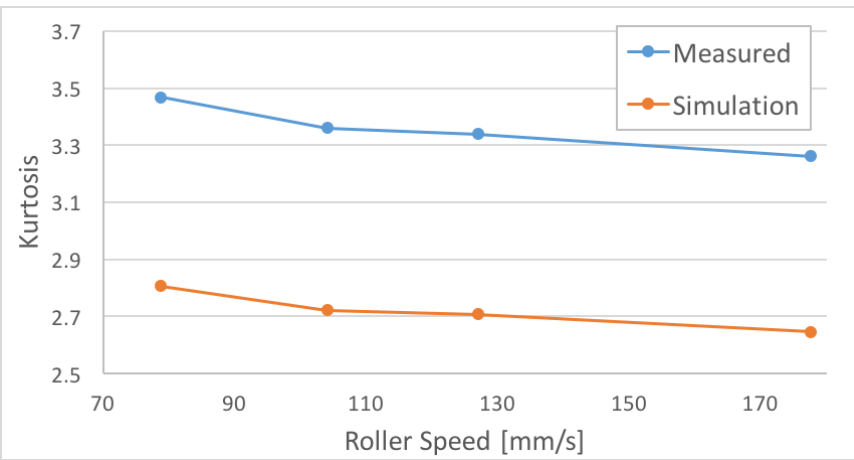

Figure 8: Kurtosis of the surface measured by the laser scanner and from simulation for different roller speeds.

Experimental measurements were acquired using a ShapeGrabber SG109 3D laser scanner which was able to scan $30 \mathrm{~mm}$ of the surface at one time with a measurement resolution of $15 \mu \mathrm{m}$. Several surface roughness measures, including the skewness and kurtosis of the surface height, were computed according to ISO 25178 [45]. Experimental and simulation results for the kurtosis of the surface distribution are shown in Fig. 8. The manifest trend is that as the roller speed increases the kurtosis decreases resulting in a rougher surface with a higher standard deviation. These preliminary results show that qualitatively the trends seen in experiments when changing the roller speed are similar to those seen in the simulation data.

\section{Conclusions}

The salient point of this contribution is that using CMBD in additive manufacturing simulation provides $(i)$ insights into how various stages of the manufacturing process work; and, (ii) sensitivities with respect to operating parameters. This information can subsequently guide efforts to increase throughput and improve part quality. Two examples were provided to substantiate this claim. In the first one, CMBD was used to enable the $3 \mathrm{D}$ printing of a dress, making the point that CMBD is useful in situations where part geometries are complex and manually determining their position/orientation inside the 3D printer volume is time consuming. The second example was a demonstration of how CMBD can be used to predict surface quality in a layering process for SLS printing.

Looking ahead, the priorities are accounting for moisture in the contact model, studying the impact of non spherical geometries in powders, and reducing simulation times in Chrono via parallel computing in order to augment the spectrum of problems that CMBD can tackle in the area of granular dynamics and powder simulation. Finally, we are investigating CMBD approaches to characterize fluid-solid interaction phenomena in manufacturing in general, and 3D printing in particular. The focus is on suspensions and polymer flows.

\section{Acknowledgments}

Financial support was provided by Army Research Office award W911NF-12-1-0395 and National Science Foundation award CMMI-GOALI-1362583.

\section{References}

[1] N. Hopkinson, P. Dickens, Emerging Rapid Manufacturing Processes, John Wiley \& Sons, Ltd, 2006, pp. 55-80. doi:10.1002/0470033991.ch5. URL http://dx.doi.org/10.1002/0470033991.ch5

[2] S. Upcraft, R. Fletcher, The rapid prototyping technologies, Assembly Automation 23 (4) (2003) 318-330. doi:10.1108/01445150310698634. URL http: //dx.doi.org/10.1108/01445150310698634

[3] V. Petrovic, J. V. H. Gonzalez, O. J. Ferrando, J. D. Gordillo, J. R. B. Puchades, L. P. Grian, Additive layered manufacturing: sectors of industrial application shown through case studies, International Journal of Production Research 49 (4) (2011) 1061-1079. doi:10.1080/00207540903479786.

URL http: //dx.doi.org/10.1080/00207540903479786

[4] E. Baur, T. A. Osswald, N. Rudolph, S. Brinkmann, E. Schmachtenberg (Eds.), Saechtling Kunststoff Taschenbuch (Plastics Handbook), 31st Edition, Carl Hanser Verlag GmbH \& Co. KG, 2013.

URL http: //amazon.de/o/ASIN/3446434429/

[5] J. P. Kruth, X. Wang, T. Laoui, L. Froyen, Lasers and materials in selective laser sintering, Assembly Automation 23 (4). doi: $10.1108 / 01445150310698652$. URL http: //hdl.handle.net/2436/29608

[6] S. L. Ford, Additive manufacturing technology: Potential implications for us manufacturing competitiveness, Journal of International Commerce and Economics.

[7] S. E. T. Corporation, Spacex launches 3d-printed part to space, creates printed engine chamber.

URL http://www.spacex.com/news/2014/07/31/spacexlaunches-3d-printed-part-space-creates-printed-enginechamber-crewed

[8] R. Kraft, T. McMahan, K. Henry, Nasa tests limits of 3-d printing with powerful rocket engine check (2013).

[9] J. J. Dunn, D. N. Hutchison, A. M. Kemmer, A. Z. Ellsworth, M. Snyder, W. B. White, B. R. Blair, 3d printing in space: enabling new markets and accelerating the growth of orbital infrastructure, Proc. Space Manufacturing 14: Critical Technologies for Space Settlement. Space Studies Institute, 29-31 October 2010, Mountain View, CA.

[10] M. M. Johnston, M. J. Werkheiser, M. P. Snyder, J. E. Edmunson, 3d printing in zero-g iss technology demonstration. 
[11] N. Aeronautics, S. Administration, Space station 3-d printer builds ratchet wrench to complete first phase of operations.

URL http://www.nasa.gov/mission_pages/station/research/ news/3Dratchet_wrench

[12] D. Drummer, D. Rietzel, F. Kühnlein, Development of a characterization approach for the sintering behavior of new thermoplastics for selective laser sintering, Physics Procedia 5, Part B (0) (2010) 533 - 542. doi:http://dx.doi.org/10.1016/j.phpro.2010.08.081.

URL http://www.sciencedirect.com/science/article/ $\mathrm{pii/S1875389210005079}$

[13] E. J. Haug, Computer-Aided Kinematics and Dynamics of Mechanical Systems Volume-I, Prentice-Hall, Englewood Cliffs, New Jersey, 1989.

[14] E. Hairer, G. Wanner, Solving Ordinary Differential Equations II: Stiff and Differential-Algebraic Problems, Springer, 1996

[15] A. A. Shabana, Dynamics of Multibody Systems, 4th Edition, Cambridge University Press, Cambridge, England., 2013.

[16] A. F. Filippov, F. M. Arscott, Differential Equations with Discontinuous Righthand Sides: Control Systems, Vol. 18, Springer, 1988.

[17] D. E. Stewart, Rigid-body dynamics with friction and impact, SIAM Review 42(1) (2000) 3-39.

[18] D. E. Stewart, J. C. Trinkle, An implicit time-stepping scheme for rigidbody dynamics with inelastic collisions and Coulomb friction, International Journal for Numerical Methods in Engineering 39 (1996) 26732691

[19] M. Anitescu, J. F. Cremer, F. A. Potra, Formulating 3D contact dynamics problems, Mechanics of Structures and Machines 24(4) (1996) 405-437.

[20] J. Trinkle, J.-S. Pang, S. Sudarsky, G. Lo, On dynamic multi-rigidbody contact problems with Coulomb friction, Zeitschrift fur angewandte Mathematik und Mechanik 77 (1997) 267-279.

[21] C. Glocker, F. Pfeiffer, An LCP-approach for multibody systems with planar friction, in: Proceedings of the CMIS 92 Contact Mechanics Int. Symposium, Lausanne, Switzerland, 2006, pp. 13-20.

[22] V. Acary, B. Brogliato, Numerical methods for nonsmooth dynamical systems: applications in mechanics and electronics, Vol. 35, Springer Science \& Business Media, 2008.

[23] D. M. Kaufman, D. K. Pai, Geometric Numerical Integration of Inequality Constrained, Nonsmooth Hamiltonian Systems, SIAM Journal on Scientific Computing 34 (5) (2012) A2670-A2703. doi:10.1137/100800105.

[24] M. Anitescu, A. Tasora, An iterative approach for cone complementarity problems for nonsmooth dynamics, Computational Optimization and Applications 47 (2) (2010) 207-235. doi:10.1007/s10589-008-9223-4.

[25] BULLET: Physics Simulation Forum, Bullet physics library, available online at http://www.bulletphysics.com/Bullet/wordpress (2010).

[26] R. L. Smith, Open Dynamics Engine, available online at http://www.ode.org/ (2015).

[27] G. T. G. Lab, Dart (Dynamic Animation and Robotics Toolkit), available online at http://dartsim.github.io/ (2015).

[28] M. a. Sherman, A. Seth, S. L. Delp, Simbody: Multibody dynamics for biomedical research, Procedia IUTAM 2 (2011) 241-261. doi:10.1016/j.piutam.2011.04.023 URL http://dx.doi.org/10.1016/j.piutam.2011.04.023

[29] H. Mazhar, T. Heyn, A. Pazouki, D. Melanz, A. Seidl, A. Bartholomew, A. Tasora, D. Negrut, Chrono: a parallel multi-physics library for rigidbody, flexible-body, and fluid dynamics, Mechanical Sciences 4 (1) (2013) 49-64. doi:10.5194/ms-4-49-2013.

[30] D. Melanz, M. Tupy, B. Smith, K. Turner, D. Negrut, On the validation of a differential variational inequality approach for the dynamics of granular material-DETC2010-28804, in: Proceedings to the 30th Computers and Information in Engineering Conference, 2010.

[31] R. Shotwell, A Comparison of Chrono::EngineÅs Primitive Joints with ADAMS Results, Tech. Rep. TR-2012-01: http://sbel.wisc.edu/ documents/TR-2012-01.pdf, Simulation-Based Engineering Laboratory, University of Wisconsin-Madison (2012). URL http://sbel.wisc.edu/documents/TR-2012-01.pdf

[32] H. Mazhar, J. Bollmann, E. Forti, A. Praeger, T. Osswald, D. Negrut, Studying the effect of powder geometry on the selective laser sintering process, in: Society of Plastics Engineers (SPE) ANTEC, 2014

[33] R. Serban, H. Mazhar, D. Melanz, P. Jayakumar, D. Negrut, A Comparative Study of Penalty and Complementarity Methods for Handling Frictional Contact in Large Multibody Dynamics Problems, in: 17th U.S. National Congress on Theoretical and Applied Mechanics (USNC-TAM),
2014.

[34] M. Taylor, R. Serban, Validation of Basic Modeling Elements in Chrono, Tech. Rep. TR-2015-05: http://sbel.wisc.edu/documents/TR2015-05.pdf, Simulation-Based Engineering Laboratory, University of Wisconsin-Madison (2015)

URL http://sbel.wisc.edu/documents/TR-2015-05.pdf

[35] A. Tasora, R. Serban, H. Mazhar, A. Pazouki, D. Melanz, J. Fleischmann, M. Taylor, H. Sugiyama, D. Negrut, Chrono: An open source multiphysics dynamics engine, in: T. Kozubek (Ed.), Lecture Notes in Computer Science, under review, Springer, 2015, pp. - .

[36] A. Hindmarsh, P. Brown, K. Grant, S. Lee, R. Serban, D. Shumaker, C. Woodward, SUNDIALS: Suite of nonlinear and differential/algebraic equation solvers, ACM Transactions on Mathematical Software (TOMS) 31 (3) (2005) 363-396.

[37] D. Negrut, R. Rampalli, G. Ottarsson, A. Sajdak, On an implementation of the Hilber-Hughes-Taylor method in the context of index 3 differential-algebraic equations of multibody dynamics (detc200585096), J. Comput. Nonlinear Dyn 2 (1) (2007) 73-85.

[38] H. Mazhar, T. Heyn, A. Tasora, D. Negrut, Using Nesterov's method to accelerate multibody dynamics with friction and contact, ACM Trans. Graph. 34 (3).

[39] M. Berzeri, M. Campanelli, A. A. Shabana, Definition of the elastic forces in the finite-element absolute nodal coordinate formulation and the floating frame of reference formulation, Multibody System Dynamics 5 (2001) 21-54

[40] A. Pazouki, D. Negrut, Direct simulation of lateral migration of buoyant particles in channel flow using GPU computing, in: Proceedings of the 32nd Computers and Information in Engineering Conference, CIE32, August 12-15, Chicago, IL, USA, American Society of Mechanical Engineers, 2012.

[41] D. Negrut, A. Tasora, H. Mazhar, T. Heyn, P. Hahn, Leveraging parallel computing in multibody dynamics, Multibody System Dynamics 27 (1) (2012) 95-117.

[42] A. Pazouki, D. Negrut, A numerical study of the effect of particle properties on the radial distribution of suspensions in pipe flow, Computers \& Fluids 108 (2015) $1-12$.

[43] Project Chrono, Chrono: An Open Source Framework for the Physics-Based Simulation of Dynamic Systems, http: //www.projectchrono.org, accessed: 2015-02-07.

[44] Blender Foundation, Blender, http: //www.blender.org/ (2014).

[45] ISO 25178. Geometric product specifications (gps) surface texture: areal, 2008 